\title{
Interventional radiotherapy as exclusive treatment for primary nasal vestibule cancer: single-institution experience
}

\author{
Luca Tagliaferri, MD, PhD!, Nadia Carra, MD², Valentina Lancellotta, MD', Davide Rizzo, MD³, Calogero Casà, MD², \\ Giancarlo Mattiucci, MD, PhD',2, Claudio Parrilla, MD4, Bruno Fionda, MD!, Francesco Deodato, MD5, \\ Patrizia Cornacchione, MSc', Maria Antonietta Gambacorta, MD, PhD 1,2, Gaetano Paludetti, MD4,6, \\ Vincenzo Valentini, MD ${ }^{1,2}$, Francesco Bussu, MD 3,7 \\ U.O.C. Radioterapia Oncologica, Dipartimento di Diagnostica per immagini, Radioterapia Oncologica ed Ematologia, Fondazione \\ Policlinico Universitario "A. Gemelli" IRCCS, Roma, Italy, ${ }^{2}$ sttituto di Radiologia, Università Cattolica del Sacro Cuore, Roma, Italy. \\ ${ }^{3}$ Divisione di Otorinolaringoiatria, Azienda Ospedaliero Universitaria, Sassari, Italy, ${ }^{4}$ U. O.C. Otorinolaringoiatria, Dipartimento Scienze \\ dell'Invecchiamento, Neurologiche, Ortopediche e della testa collo, Fondazione Policlinico Universitario "A. Gemelli" IRCCS, Roma, Italy, \\ ${ }^{5}$ Dipartimento di Radioterapia, Fondazione Ricerca e Cura Giovanni Paolo II, Università Cattolica del Sacro Cuore, Campobasso, Italy. \\ ${ }^{6}$ Istituto di Otorinolaringoiatria, Università Cattolica del Sacro Cuore, Roma, Italy, 'Otorinolaringoiatria, Università degli studi di Sassari, \\ Sassari, Italy
}

\begin{abstract}
Purpose: The aim of this paper was to evaluate treatment outcomes following interventional radiotherapy (brachytherapy - BT) for nasal vestibule cancer.

Material and methods: Considering histological diagnosis and staging, a multidisciplinary tumor board indicated an exclusive interventional radiotherapy for all patients. Plastic tubes were placed mainly with interstitial approach. The total dose was 44 Gy in 14 fractions, 3 Gy/fraction (except for the first and last fractions, 4 Gy), 2 fractions per day (b.i.d.), 5 days a week. Inclusion criteria for this analysis were: patients affected by squamous cell carcinoma with follow-up more than 6 months.

Results: 20 patients with primary nasal vestibule cancer were treated with IRT from May 2012 to June 2019. We excluded 4 patients due to follow-up less than 6 months and 2 patients affected by basal cell carcinoma. In total, 14 consecutive previously untreated patients were considered for definitive analysis, median age was 67.5 (range, 51-83) years, median follow-up was 53 (range, 6-84) months. All patients followed the protocol except one, who received a total dose of $42 \mathrm{~Gy}$ in 12 fractions, 3 Gy per 6 fractions, and 4 Gy per 6 fractions. Local control at 12, 24, and 36 months was $85.7 \%$. Overall survival at 12 months was $92.3 \%$, at 24 months was $76.9 \%$, and at 36 months was $69.2 \%$. Staging system proposed by Wang was statistically significant on local control (LC), disease-free survival (DFS), disease-specific survival (DSS), and overall survival (OS). Excellent cosmetic results were observed.

Conclusions: This study confirms that interventional radiotherapy could be considered as a definitive treatment in nasal vestibule cancer with excellent oncological and cosmetic outcomes.

Key words: interventional radiotherapy, brachytherapy, nasal vestibule cancer, PRO, patient-reported outcomes.

\section{Purpose}

Squamous cell carcinoma (SCC) of the nasal vestibule is a rare type of tumor ( $1 \%$ of head and neck cancers) [1] that can impact patients' quality of life, since the therapy may be often associated with unaesthetic results. Radiation therapy and surgery are frequently proposed therapeutic modalities, but no consensus exists regarding the best approach and staging system. For this reason, the best strategy should be discussed in a multidisciplinary setting. Some clinicians prefer AJCC/TNM classification [2], while others use the staging system proposed by Wang [3], which is simple and includes only three stages (Table 1).

Even though no guidelines exist to provide a uniform treatment indication, radiotherapy can be considered as
Address for correspondence: Nadia Carra, MD, Fondazione Policlinico Universitario Agostino Gemelli IRCCS - Università Cattolica del Sacro Cuore, Istituto di Radiologia, Largo Agostino Gemelli 8, Roma, Italy, phone: +39-34-76974405, e-mail: nadia.carra@yahoo.it
Received: 24.10 .2019

Accepted: 12.08 .2020

Published: 30.10 .2020 
Table 1. Wang's staging system

\begin{tabular}{ll} 
Category & \multicolumn{1}{c}{ Definition } \\
\hline T1 & $\begin{array}{l}\text { Limited to the nasal vestibule, relative superfi- } \\
\text { cial, and involve } 1 \text { or more sites within the nasal } \\
\text { vestibule }\end{array}$ \\
\hline T2 & $\begin{array}{l}\text { Extended from the nasal vestibule to the adjacent } \\
\text { structures, such as the upper nasal septum, upper } \\
\end{array}$ \\
& $\begin{array}{l}\text { lip, philtrum, skin of the nose, and/or nasolabial } \\
\text { fold, but they are not fixed to the underlying bone }\end{array}$ \\
\hline T3 & $\begin{array}{l}\text { cogingival sulcus, large portion of the upper lip, } \\
\text { upper septum, turbinate, and/or paranasal sinus, } \\
\text { fixed with deep muscle or bone involvement }\end{array}$
\end{tabular}

exclusive therapy in T1-T2 tumors or in combination with surgery in locally advanced tumors $[4,5,6,7]$.

Radiation therapy can be delivered as external beam radiotherapy (EBRT), interventional radiotherapy (IRT), or combination of both. IRT consists of placing radioactive sources within or directly adjacent to a tumor, which is a way of delivering highly targeted and conformal radiation [8], with excellent sparing of the surrounding tissues [9]. IRT can be considered as an excellent approach and, in some cases, it shows better tumor control and cosmesis than EBRT $[10,11]$ with higher degree of patients' satisfaction than surgery [12].

The present paper reports the results (local and regional control, disease-free survival, overall survival, disease specific survival) of patients included in our study. Moreover, it demonstrates the dosimetric data and patients' satisfaction of a single-institution analysis of 14 patients treated with IRT.

\section{Material and methods}

All consecutive patients, affected by primary nasal vestibule cancer, treated at the Interventional Oncology Center (IOC) of the Gemelli-ART (advanced radiation therapy) with IRT from May 2012 to June 2019 were evaluated. Squamous cell carcinoma with follow-up more than 6 months were the inclusion criteria for this analysis.

Twenty patients diagnosed with primary nasal vestibule cancer were treated. However, for statistical analysis, 4 patients were not considered due to follow-up time less than 6 months, and 2 patients affected by basal cell carcinoma. Therefore, for definitive analysis, 14 patients with SCC were investigated.

All patients were evaluated by a multidisciplinary head and neck oncology team, and were subjected to physical examination, biopsy of the primary lesion for histologic confirmation, and computed tomography (CT) or magnetic resonance (MRI) for staging. All patients signed an informed consent both for the procedure and for the treatment as well as for a possible use of personal data in scientific papers. For T classification, the staging system proposed by Wang [3] was adopted. Toxicities were assessed using the common terminology criteria for adverse events (CTCAE) version 4.0 [13]. Patients' characteristics are reported in Table 2.
Table 2. Patients' characteristics

\begin{tabular}{lc} 
Factors & $n(\%)$ \\
\hline Sex & $10(71.4)$ \\
\hline Male & $4(28.6)$ \\
\hline Female & $67.5($ range, 51-83) years \\
\hline Age, median & $3(21.4)$ \\
\hline 1 & $10(71.4)$ \\
\hline 3 & $1(7.2)$ \\
\hline Initial N & $12(85.7)$ \\
\hline N- & $2(14.3)$ \\
\hline N+ & $53($ range, 6-84) months
\end{tabular}

Two patients presented with clinical nodal involvement. No irradiation, but modified neck dissection was performed, which confirmed pathological involvement; one patient had already been irradiated for Hodgkin lymphoma, and one followed only clinical and radiological follow-up after multidisciplinary discussion.

In the absence of definitive literature data and consensus regarding assessment methods, the patients were requested to express a subjective opinion on their cosmetic outcome. In order to obtain an easy-to-use method to calculate patients' satisfaction with cosmetic results, a scale ranging from 1 to 4 was used. The scale consisted of satisfaction staging, with 1 meaning very dissatisfied, 2 - dissatisfied, 3 - satisfied, and 4 - very satisfied.

\section{Implant procedure}

Flexible tubes of $6 \mathrm{~F}$ were implanted, with $0.8-1.2 \mathrm{~cm}$ spacing, according to the Paris system rules to adequately cover the clinical target volume (CTV) and $0.5-1 \mathrm{~cm}$ margins if possible (due to anatomical limitations). The implantation was performed keeping the tubes fully interstitial, and avoiding piercing the perichondrium and cartilages to prevent septal and alar perforation, unless clinically necessary due to tumor infiltration.

Local fixation was done with buttons sutured to the skin and, if needed, with Merocel packing (Medtronic Inc., Minneapolis, MN, USA). The number of catheters varied according to the size of the target. In some cases, during implantation, endoscopic vision with $0-45^{\circ}$ endoscopes was used.

After the plastic tubes were implanted, all patients underwent $\mathrm{CT}$, and $\mathrm{CTV}$ and catheters were reconstructed. Treatment planning was implemented with Oncentra Brachy (Elekta, Stockholm, Sweden) and the treatment was delivered using high-dose-rate (HDR) afterloader (Elekta MicroSelectron or Flexitron). The treatment protocol was prescribed with a total dose of $44 \mathrm{~Gy}$ 
in 14 fractions, 3 Gy per fraction (except the first and last ones, 4 Gy), 2 fractions per day (b.i.d.), 5 days per week $\left(\mathrm{EQD}_{2} 48.5 \mathrm{~Gy}\right.$ and BED $58 \mathrm{~Gy}$ considering the tumor $\mathrm{a} / \beta$ ratio of 10) for an overall treatment time of 9 days. All patients followed the prescription protocol but one who received a total dose of $42 \mathrm{~Gy}$ in 12 fractions, 3 Gy per 6 fractions, and 4 Gy per 6 fractions (EQD 251.5 Gy and BED 61.8 Gy considering the tumor $\alpha / \beta$ ratio of 10 ). Images of a brachytherapy (BT) implant and the treatment plan are presented in Figure 1.

\section{Dosimetric criteria}

We considered the range and median of $\mathrm{V}_{200}, \mathrm{~V}_{150}$, $\mathrm{V}_{100}, \mathrm{~V}_{90}$, and $\mathrm{V}_{85}$, which represent the clinical target volume (CTV) covered by $200 \%, 150 \%, 100 \%, 90 \%$, and $85 \%$ of the prescription dose, respectively. Moreover, the median and range of dose non-uniformity ratio (DNR), dose homogeneity index (DHI), coverage index (CI), and overdose volume index (ODI) were calculated.

\section{Statistical analysis}

Kaplan-Meier method was used to estimate the probability of local control (LC), regional control (RC), disease- free survival (DSF), disease-specific survival (DSS), and overall survival (OS). A univariate analysis was carried out considering the following outcomes: age ( $>65$ years old), Wang's staging, $\mathrm{V}_{100 \mathrm{cc}}$ and tumor volume.

\section{Results}

In total, 14 patients were considered for analysis. The median follow-up time was 53 (range, 6-84) months. No interruptions of the IRT schedule for acute toxicity were recorded. The mean CTV volume was $16.63 \mathrm{cc}$ (range, 4.3-40), and the median number of catheters used was 7 (range, 5-18). The median $\mathrm{V}_{100}$ was $11.77 \mathrm{cc}$ and the mean $\mathrm{V}_{100}$ was $14.28 \mathrm{cc}$, with a range between 2.79 and 37.98. The univariate analysis performed for the $\mathrm{V}_{100}$ did not show any statistically significant correlation. Dosimetric data are reported in Table 3.

Two local recurrences were observed, including one marginal and one central recurrences. One of these patients died from the disease (according to Wang's staging, the pretreatment staging of one patient was T2, whereas the other was T3, they were both N0). Two regional recurrences in follow-up were recorded, and both patients were salvaged with neck dissection and adjuvant external beam
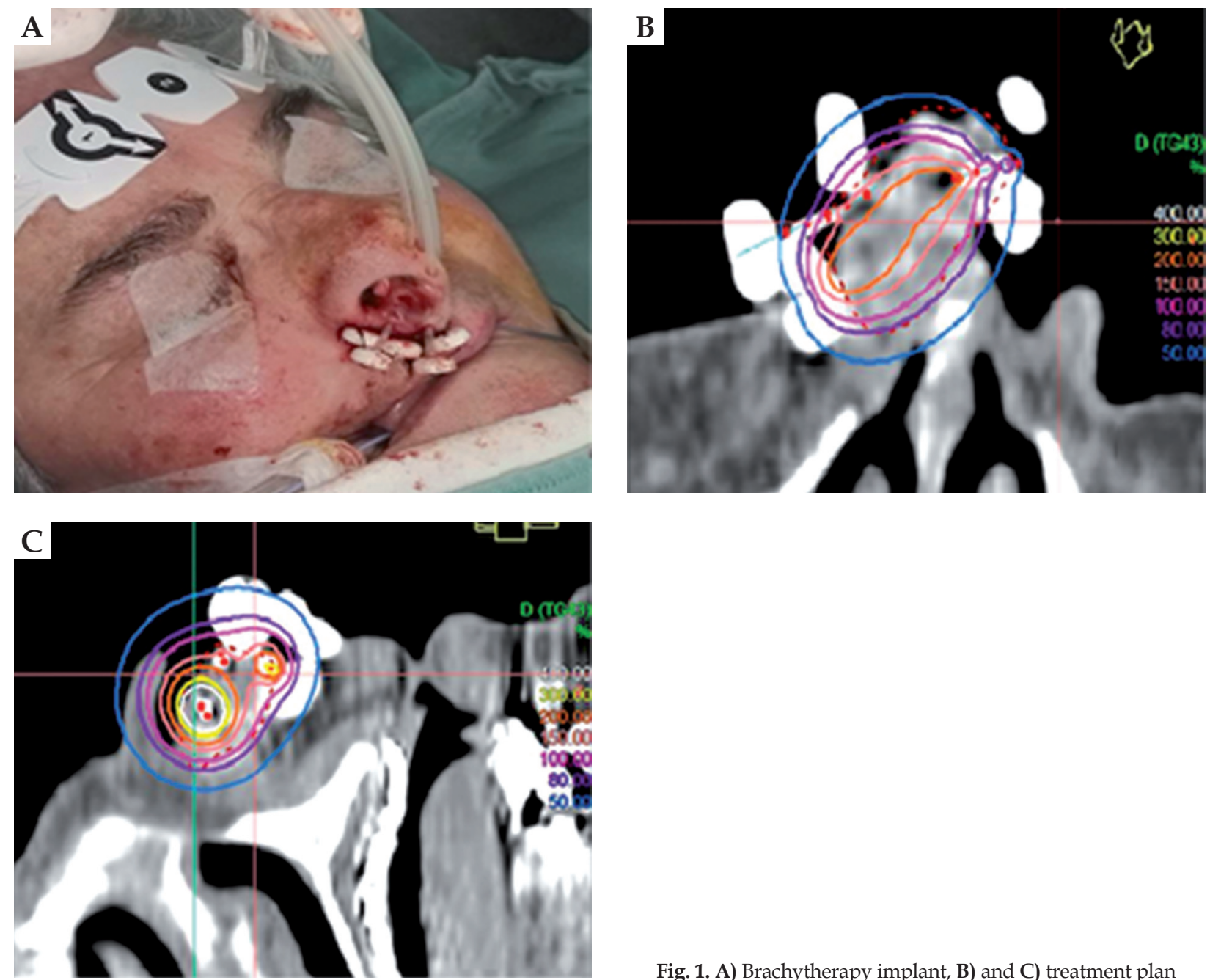

Fig. 1. A) Brachytherapy implant, B) and C) treatment plan 
Table 3. Dosimetric data

\begin{tabular}{llll} 
& Mean & Median & Range \\
\hline $\mathrm{V}_{200}(\%)$ & 28.874 & 29.310 & $10.828-53.375$ \\
\hline $\mathrm{V}_{200}(\mathrm{cc})$ & 4.930 & 3.250 & $0.620-21.350$ \\
\hline $\mathrm{V}_{150}(\%)$ & 52.244 & 51.345 & $26.977-89.803$ \\
\hline $\mathrm{V}_{150}(\mathrm{cc})$ & 9.244 & 6.885 & $1.160-30.330$ \\
\hline $\mathrm{V}_{100}(\%)$ & 84.417 & 86.509 & $64.884-96.951$ \\
\hline $\mathrm{V}_{100}(\mathrm{cc})$ & 14.275 & 11.770 & $2.790-37.980$ \\
\hline $\mathrm{V}_{90}(\%)$ & 89.736 & 92.283 & $74.651-98.066$ \\
\hline $\mathrm{V}_{90}(\mathrm{cc})$ & 15.076 & 12.405 & $3.210-38.980$ \\
\hline $\mathrm{V}_{85}(\%)$ & 91.919 & 94.320 & $78.311-98.649$ \\
\hline $\mathrm{V}_{85}(\mathrm{cc})$ & 15.399 & 12.265 & $3.430-39.290$ \\
\hline $\mathrm{CTV}(\mathrm{cc})$ & 16.633 & 13.155 & $4.300-40.000$ \\
\hline $\mathrm{Cl}$ & 0.844 & 0.865 & $0.649-0.970$ \\
\hline ODI & 0.332 & 0.330 & $0.160-0.562$ \\
\hline $\mathrm{DNR}$ & 0.605 & 0.589 & $0.416-0.926$ \\
\hline $\mathrm{DHI}$ & 0.395 & 0.411 & $0.074-0.584$ \\
\hline $\mathrm{CIV}-\mathrm{cin})$ & & & \\
\hline
\end{tabular}

$C T V$ - clinical target volume, $\mathrm{Cl}$ - coverage index, ODI-overdose volume index $D N R$ - dose non-uniformity ratio, DHI - dose homogeneity index

radiotherapy (according to Wang's staging, the pretreatment staging of one patient was $\mathrm{T} 2$, whereas the other was T1, they were both N0). Toxicity was monitored during follow-up (both acute and late), and no G3 or G4 toxicities were observed. G1/G2 toxicity were present in 4 patients, with edema and crusts as the most commonly recorded.

Local control at 12, 24, and 36 months were $85.7 \%$ (Figure 2). Regional control (RC) at 12 and 24 months were $92.9 \%$, and $81.2 \%$ at 36 months (Figure 3). DFS at 12 and 24 months were $78.6 \%$, and $67.3 \%$ at 36 months (Figure 4 ). OS at 12 months was $92.3 \%$, at 24 months was $76.9 \%$, and at 36 months was $69.2 \%$ (Figure 5). Disease-specific survival at 12, 24, and 36 months were 92.3\% (Figure 6).

From the univariate analysis performed, only the staging system proposed by Wang showed a statistically significant $(p$-value $<0.05)$ correlation with oncological endpoints (LC, DSF, DSS, and OS) (Table 4).

Very good cosmetic results were observed; of the eight patients interviewed, seven patients (87.5\%) expressed a good degree of satisfaction and only one patient $(12.5 \%)$ was not completely satisfied (this patient had both $\mathrm{V}_{150}$ and $\mathrm{V}_{200}$ higher than the median). Patients' satisfaction in detail is reported in Figure 7. In Figure 8, two pictures of the same patient before and after the treatment are displayed.

\section{Discussion}

The choice of the most appropriate therapeutic approach and staging system for nasal vestibule cancer is a matter of growing interest in the head and neck oncolog-

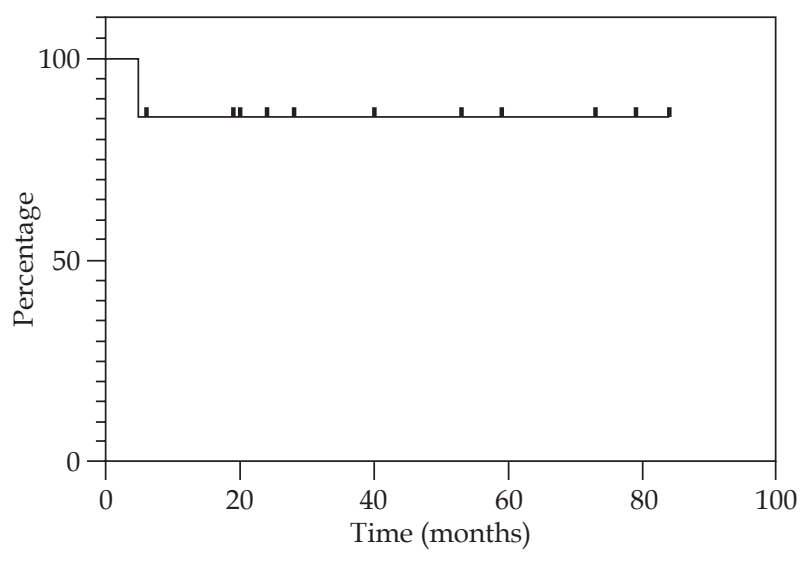

Fig. 2. Kaplan-Meier graph presenting the cumulative proportion of local control

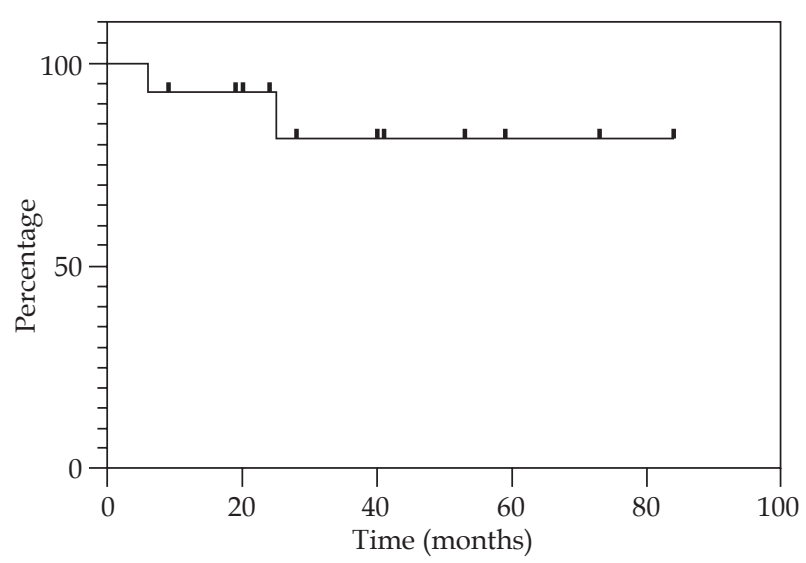

Fig. 3. Kaplan-Meier graph presenting the cumulative proportion of regional control



Fig. 4. Kaplan-Meier graph presenting the cumulative proportion of disease-free survival

ical community. Based on the AJCC/International Union Against Cancer classification, nasal vestibule cancer is considered in the frame of nasal cavity tumors [2] and the disease is defined as cT4a in case of skin involvement, while bone invasion can be found also in cT1. However, the invasion of the skin does not drastically impact the 


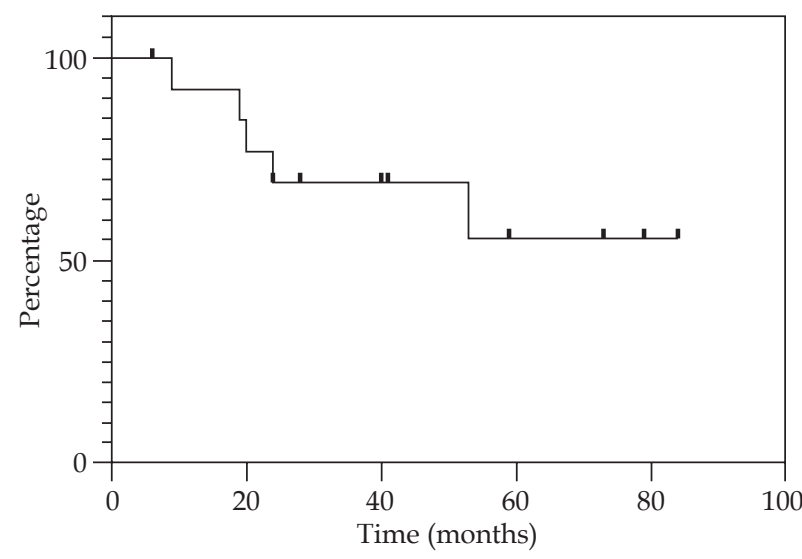

Fig. 5. Kaplan-Meier graph presenting the cumulative proportion of overall survival

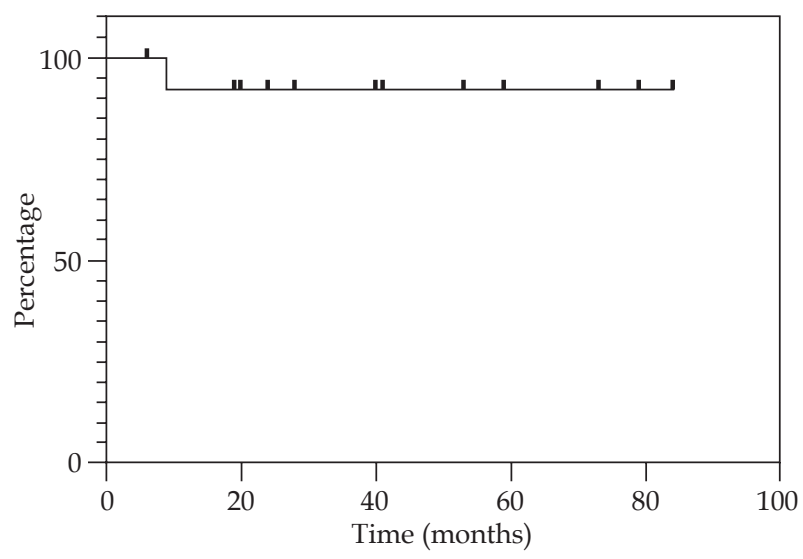

Fig. 6. Kaplan-Meier graph presenting the cumulative proportion of disease-specific survival

Table 4. Outcomes of statistical univariate analysis using log-rank test for DFS, DSS, OS, LC, and RC

\begin{tabular}{lccccc} 
& DFS & DSS & OS & LC (T) & RC (N) \\
& $p$-value & $p$-value & $p$-value & $p$-value & 0.790 \\
\hline Wang's staging & 0.050 & 0.002 & 0.001 & 0.046 & 0.920 \\
\hline Age (> 65 years) & 0.760 & 0.360 & 0.170 & 0.830 & 0.585 \\
\hline Tumor volume & 0.402 & 1.000 & 0.835 & 0.998 & 0.409
\end{tabular}

DFS - disease-free survival, DSS - disease-specific survival, OS - overall survival, LC - local control, RC - regional control

prognosis, unlike bone invasion [14]. For these reasons, for T classification, we adopted Wang's staging system, specific for nasal vestibule cancer, which has been shown to better predict prognosis $[15,16]$. In the present series, in accordance with these considerations, the Wang's staging system represents a statistically significant prognostic factor in terms of LC, DSF, DSS, and OS.

No significant differences between patients primarily treated by surgery and IRT in terms of survival and loco-regional control have been defined [12]. Nowadays, IRT is recognized as a valid option for treatment of this tumor $[17,18]$. Indeed, surgery and radiotherapy (IRT or external beams) may provide similar chances for the cure of nasal vestibule cancer. In such a situation, as always more often in modern head and neck oncology, the issues concerning quality of life of patients become decisive for the treatment recommendation.

The quality of life of patients successfully treated for nose vestibule cancer and therefore their reported satisfaction are affected mainly by 2 parameters: firstly, the aesthetic outcome and secondly, the preservation of specific nose functions (e.g., breathing, smell, air filtering, mucociliary clearance).

Aesthetic outcome is critical because the nasal vestibule is probably the most exposed and noticed part of the whole body, where minimal imperfections, scars, or deformity have the highest esthetical and social impact. Moreover, surgical reconstruction of full thickness defect (which is the most frequent condition with nearly constant involvement of the skin) is extremely difficult [19].

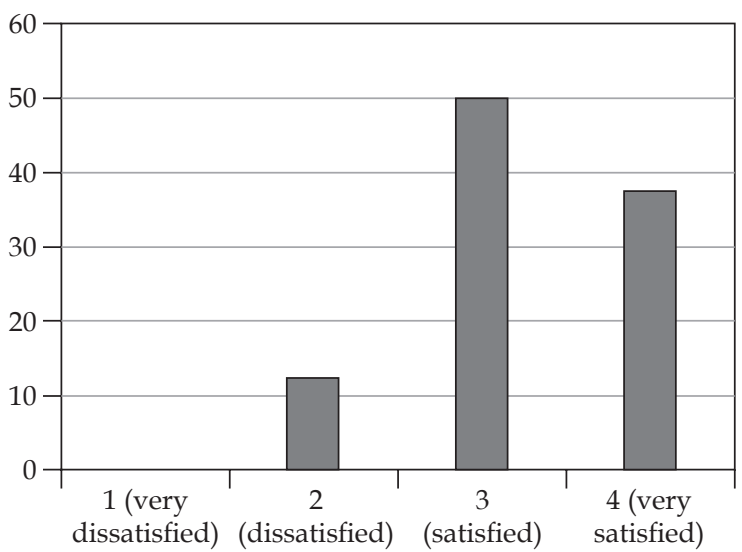

Fig. 7. Patients' satisfaction

It is important to note that in the present study, the patient who was not completely satisfied with the cosmetic result had both $\mathrm{V}_{150}$ and $\mathrm{V}_{200}$ more than the median. However, no statistically significant correlation was found in our study between clinical outcomes and dosimetric data.

Since reliefs and hollows formed by cartilages are practically impossible to reproduce, both by local and free flaps, various authors [20] believe that anchored prostheses may be the best choice when performing total rhinectomy. Therefore, the anatomy preservation represents the best approach with regard to the degree of 

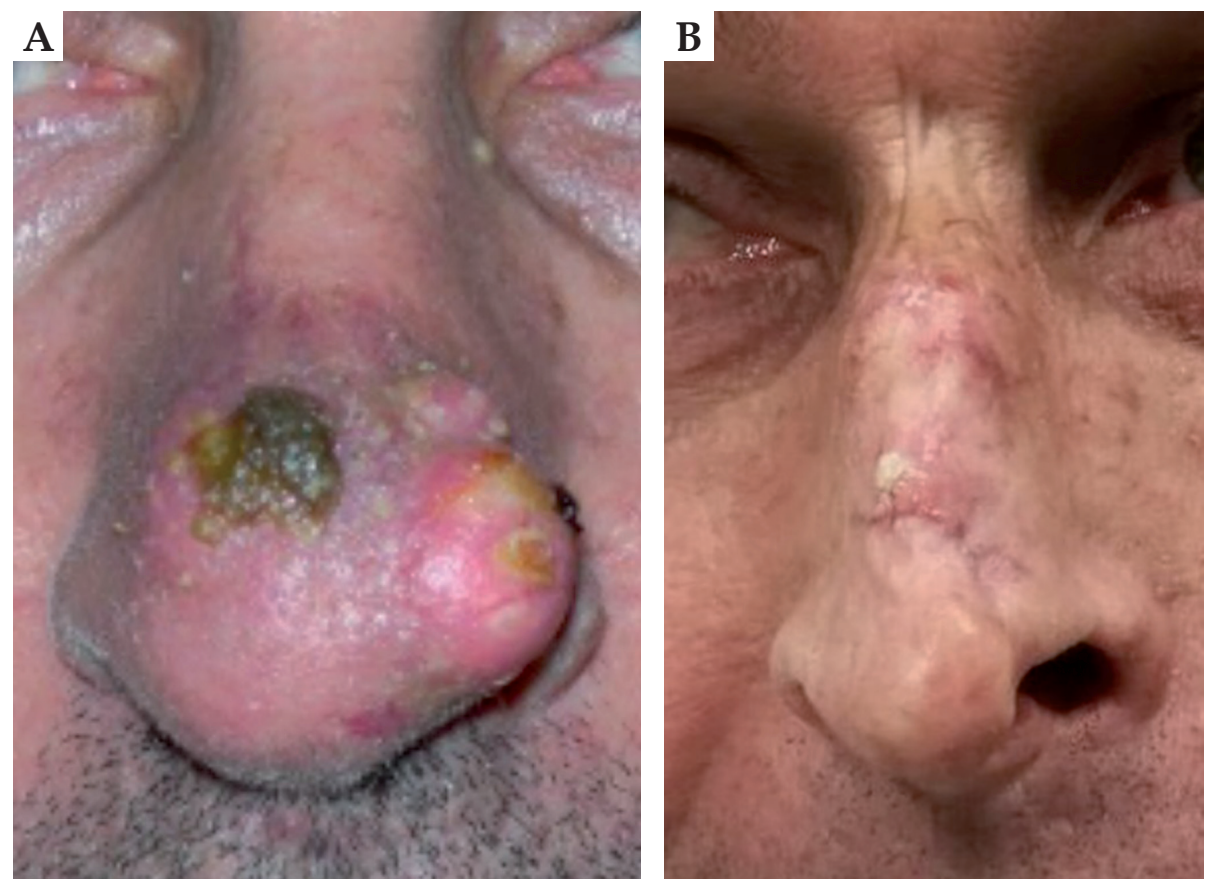

Fig. 8. A) Patient before the treatment, B) the same patient after the treatment

satisfaction, as observed from patients' assessments [12], which is also shown in the present study. IRT can also be used successfully in the elderly where comprehensive surgery, especially repeated reconstructive surgical approaches, can more often be contraindicated.

In the literature, commonly mentioned functional side effects are nasal dryness, crusts, and adhesions [21]. In our study, no G3/G4 acute toxicity was recorded and all the patients completed the treatment.

The principal limitation of this study was a relatively small number of patients and a brief follow-up of some patients. The implementation of randomized trials is recommended but it is difficult, considering the rarity of nasal vestibule cancer. However, systems, which allow sharing of data among different institutions are already present in daily practice for head and neck patients, especially for rare tumors treated with IRT [22].

To date, the largest cohort of patients was observed by Czerwinski et al. In more than 100 consecutive patients, brachytherapy was used as a sole treatment for nasal vestibule carcinoma. They concluded that BT offers excellent local control for Wang T1-T2 tumors with patients' high level of satisfaction [23].

Even though studies like the one by Czerwinski et al. offer valuable data on this very rare disease, we believe that the use of a large database may be a good alternative. A multicentric system could help to develop predictive models and decision support tools, which could be implemented in the clinical practice to support and improve multidisciplinary discussion [24]. Moreover, it could assist in identification of patients who could benefit from this kind of treatment. According to this, we recommend a multidisciplinary approach, developing large multicentric database, and introducing this approach at an educational level [25].

\section{Conclusions}

This study confirms that IRT, in view of the safety, provides excellent tumor control and high patient's esthetic satisfaction, and could be considered as a definitive treatment in nasal vestibule cancer. However, further prospective analysis of a multinational cohort of large databases is needed to provide a more detailed view of a longterm results.

\section{Acknowledgements}

The authors would like to thank the reviewers and the section editor for the comments and suggestions received.

\section{Disclosure}

The authors report no conflict of interest.

\section{References}

1. Vital D, Morand G, Huber GF et al. Outcome in squamous cell carcinoma of the nasal vestibule: a single center experience. Head Neck 2015; 37: 46-51.

2. Amin MB, Edge SB, Greene FL et al. (Eds.). AJCC Cancer Staging Manual. 8th ed. Springer, New York 2017.

3. Wang CC. Treatment of carcinoma of the nasal vestibule by irradiation. Cancer 1976; 38: 100-106.

4. Kummer E, Rasch CR, Keus RB et al. T stage as prognostic factor in irradiated localized squamous cell carcinoma of the nasal vestibule. Head Neck 2002; 24: 268-273.

5. Johansen LV, Hjelm-Hansen M, Andersen AP. Squamous cell carcinoma of the nasal vestibule. Treatment results. Acta Radiol Oncol 1984; 23: 189-192.

6. Langendijk JA, Poorter R, Leemans CR et al. Radiotherapy of squamous cell carcinoma of the nasal vestibule. Int J Radiat Oncol Biol Phys 2004; 59: 1319-1325. 
7. Wallace A, Morris CG, Kirwan J et al. Radiotherapy for squamous cell carcinoma of the nasal vestibule. Am J Clin Oncol 2007; 30: 612-616.

8. Lukens JN, Gamez M, Hu K et al. Modern brachytherapy. Semin Oncol 2014; 41: 831-847.

9. Skin tumours. In: Practical Handbook of Brachytherapy. UIN-MED Verlag 2014.

10. Baris G, Visser AG, van Andel JG. The treatment of squamous cell carcinoma of the nasal vestibule with interstitial iridium implantation. Radiother Oncol 1985; 4: 121-125.

11. Chassagne D, Wilson JF. Brachytherapy of carcinomas of the nasal vestibule. Int J Radiat Oncol Biol Phys 1984; 10: 761.

12. Bussu F, Tagliaferri L, Mattiucci G et al. Comparison of interstitial brachytherapy and surgery as primary treatments for nasal vestibule carcinomas. Laryngoscope 2016; 126: 367-371.

13. Cancer Therapy Evaluation Program. Common Terminology criteria for Adverse Events, Version 4.0; 2009. http://ctep. cancer.gov (accessed May 2019).

14. Jeannon JP, Riddle PJ, Irish J et al. Prognostic indicators in carcinoma of the nasal vestibule. Clin Otolaryngol 2007; 32: 19-23.

15. Agger A, Von BC, Madsen AR et al. Squamous cell carcinoma of the nasal vestibule 1993-2002: a nationwide retrospective study from DAHANCA. Head Neck 2009; 31: 1593-1599.

16. Levendag PC, Nijdam WM, van Moolenburgh SE et al. Interstitial radiation therapy for early-stage nasal vestibule cancer: a continuing quest for optimal tumor control and cosmesis. Int J Radiat Oncol Biol Phys 2006; 66: 160-169.

17. Nag S, Cano ER, Demanes DJ et al. American Brachytherapy S. The American Brachytherapy society recommendations for high-dose-rate brachytherapy for head-and-neck carcinoma. Int J Radiat Oncol Biol Phys 2001; 50: 1190-1198.

18. Kovacs G, Martinez Monge R, Budrukkar A et al. GEC-ESTRO ACROP recommendations for head \& neck brachytherapy in squamous cell carcinomas: 1st update - improvement by cross sectional imaging based treatment planning and stepping source technology. Radiother Oncol 2017; 122: 248-254.

19. Tagliaferri L, Bussu F, Fionda B et al. Perioperative HDR brachytherapy for reirradiation in head and neck recurrences: single-institution experience and systematic review. Tumori 2017; 103: 516-524.

20. Goepfert H. The vex and fuss about nasal vestibule cancer. Head Neck 1999; 21: 383-384.

21. Evensen JF, Jacobsen AB, Tausjø JE, Brachytherapy of squamous cell carcinoma of the nasal vestibule. Acta Oncol 1996; 35 Suppl 8: 87-92.

22. Tagliaferri L, Budrukkar A, Lenkowicz J et al. ENT COBRA ONTOLOGY: the covariates classification system proposed by the Head \& Neck and Skin GEC-ESTRO Working Group for interdisciplinary standardized data collection in head and neck patient cohorts treated with interventional radiotherapy (brachytherapy). J Contemp Brachytherapy 2018; 10: 260-266.

23. Czerwinski MD, van Leeuwen RGH, Kaanders JHAM et al. Image guided brachytherapy for cancer of the nasal vestibule: local control and cosmesis. Int J Radiat Oncol Biol Phys 2019; 103: 913-921.

24. Tagliaferri L, Fionda B, Bussu F et al. Interventional radiotherapy (brachytherapy) for squamous cell carcinoma of the nasal vestibule: a multidisciplinary systematic review. Eur J Dermatol 2019; 29: 417-421.

25. Tagliaferri L, Vavassori A, Lancellotta V et al. Can brachytherapy be properly considered in the clinical practice? Trilogy project: The vision of the AIRO (Italian Association of Radiotherapy and Clinical Oncology) Interventional Radiotherapy study group. J Contemp Brachytherapy 2020; 12: 84-89. 